\title{
Asthma Care Quality, Language, and Ethnicity in a Multi-State Network of Low-Income Children
}

\author{
John Heintzman, MD, MPH, Jorge Kaufmann, ND, MS, Jennifer Lucas, PhD, \\ Shakira Suglia, PhD, Arvin Garg, MD, Jon Puro, MS, Sophia Giebultowicz, MA, \\ David Ezekiel-Herrera, MS, Andrew Bazemore, MD, and Miguel Marino, PhD
}

Introduction: Prior research has documented disparities in asthma outcomes between Latino children and non-Hispanic whites, but little research directly examines the care provided to Latino children over time in clinical settings.

Metbods: We utilized an electronic health record-based dataset to study basic asthma care utilization (timely diagnosis documentation and medication prescription) between Latino (Spanish preferring and English preferring) and Non-Hispanic white children over a 13-year study period.

Results: In our study population $(n=37,614)$, Latino children were more likely to have Medicaid, be low income, and be obese than non-Hispanic white children. Latinos (Spanish preferring and English preferring) had lower odds than non-Hispanic whites of having their asthma recorded on their problem list on the first day the diagnosis was noted (odds ratio $[\mathrm{OR}]=0.83$; $95 \% \mathrm{CI}, 0.77$ to 0.89 Spanish preferring; $\mathrm{OR}=0.93 ; 95 \% \mathrm{CI}, 0.87$ to 0.99 English preferring). Spanish-preferring Latinos had higher odds of ever receiving a prescription for albuterol $(\mathrm{OR}=1.96 ; 95 \% \mathrm{CI}, 1.52$ to 2.52$)$, inhaled corticosteroids $(\mathrm{OR}=1.45 ; 95 \% \mathrm{CI}, 1.01$ to 2.09$)$, or oral steroids $(\mathrm{OR}=1.48 ; 95 \% \mathrm{CI}, 1.07$ to 2.04$)$ than non-Hispanic whites. Among those with any prescription, Spanish-preferring Latinos had higher rates of albuterol prescriptions compared with non-Hispanic whites (adjusted rate ratio [aRR] $=1.0 ; 95 \% \mathrm{CI}, 1.01$ to 1.13).

Conclusions: In a multi-state network of clinics, Latino children were less likely to have their asthma entered on their problem list the first day it was noted than non-Hispanic white children, but otherwise did not receive inferior care to non-Hispanic white children in other measures. Further research can examine other parts of the asthma care continuum to better understand asthma disparities. (J Am Board Fam Med 2020;33:707-715.)

Keywords: Asthma, Electronic Health Records, Health Care Disparities, Hispanic Americans, Poverty, PracticeBased Research, Primary Health Care

\section{Introduction}

Approximately 8\% of children in the United States have asthma. ${ }^{1}$ Prior studies have demonstrated persistent disparities in the asthma care utilized by

This article was externally peer reviewed.

Submitted 27 December 2019; revised 7 March 2020; accepted 11 March 2020.

From the Department of Family Medicine, Oregon Health \& Science University, Portland (JH,JK, JL, DEH, MM); OCHIN Inc., Portland, OR (JH, JP, SG); Emory University, Atlanta, GA (SS); American Board of Family Medicine, Lexington, KY (AB); Boston Medical College, Boston, MA(AG).

Funding: National Institute on Minority Health and Health Disparities R01MD011404 (JH).

Conflicts of interest: None.

Disclosures: None

Corresponding author: John Heintzman, MD, MPH, Department of Family Medicine, Oregon Health \& Science University, 3181 SW Sam Jackson Park Road, Portland, OR 97239 (E-mail: heintzma@ohsu.edu).
Latino children compared with non-Hispanic white children, $^{2-12}$ in areas of medication use, ${ }^{2,3,6,8,9}$ asthma self-management, ${ }^{4}$ and overall access to care. $^{7,10-12}$ Asthma has longstanding national standards to guide disease management, ${ }^{13}$ including documentation of asthma as a chronic condition in the medical chart (including its severity) ${ }^{13-15}$ and appropriate prescription of medications (eg, bronchodilators and inhaled and oral corticosteroids) to match disease severity. ${ }^{13}$ Understanding disparities in the primary care environment (where these guidelines are implemented) is crucial; however, existing studies have produced conflicting findings. Studies using some surveys have found that Latino patients are less likely to have inhaled corticosteroids prescribed, $3,6,8$ while a study using another demonstrated no significant differences in these medication prescriptions. ${ }^{16}$ 
Surveys have shown limitations when used to measure health care services received over time. ${ }^{17-21}$ Since many low-income children receive their asthma care in community health centers $(\mathrm{CHCs}),{ }^{22}$ examining a $\mathrm{CHC}$ population is essential to understanding asthma care for this particular demographic. Our objective was to use a multi-state $(n=21)$ electronic health record (EHR)-based data set that serves a network of CHCs, containing greater than 37,000 children with asthma, to study differences in basic asthma care utilization (timely diagnosis documentation and medication prescription) between Latino and Non-Hispanic white children over a thirteenyear study period. We hypothesized that Latino children would have less frequent documentation of asthma-including severity-on their problem list, and lower medication prescriptions overall compared with non-Hispanic white children with asthma.

\section{Methods}

\section{Data Sources}

We utilized data from the Accelerating Data Value Across a National Community Health Center Network (ADVANCE) Clinical Research Network. ${ }^{23}$ The ADVANCE Clinical Research Network contains EHR data from the OCHIN (not an acronym) network and Health Choice Network (HCN) of CHCs in 21 states.

\section{Population}

Our study population included any child aged 3 to 17 years, ${ }^{24}$ with a primary care office visit between January 1, 2012 and January 31, 2017 and with any diagnosis code (primary or otherwise) of asthma either during a clinic visit or recorded on their problem list (as these are the possible locations for a provider to designate an asthma diagnosis code). Our diagnoses were not compared with a diagnostic standard (such as pulmonary function testing): our goal was instead to measure the care quality of those children whom the clinic designated as having asthma at some point. For the children who met this 2012 to 2017 inclusion criteria, we then had access to all EHR data as far back as 2005.

\section{Exclusion Criteria}

Patients were excluded if they had a diagnosis of cystic fibrosis and if they did not have a valid body mass index (BMI) on the date of their asthma diagnosis as BMI is an important confounder of asthma course..$^{25,26}$

\section{Dependent Variables}

Outcome variables were features of common asthma care measurable in discrete fields in the EHR. We considered 2 sets of outcomes: the accurate and timely diagnosis of asthma as a chronic condition, and the annual prescription rates of common asthma medications. Our first outcome was a binary indicator denoting whether asthma was documented on the problem list on the same day as the initial asthma diagnosis in the EHR. We utilized the recording of a chronic illness on a chart's problem list not as a standard of diagnosis, but as a fundamental, guideline driven quality care step in managing any chronic condition. ${ }^{14,15,27-29}$ Specifically, it is standard in chronic disease to document chronic conditions in some kind of registry. ${ }^{14,15}$ The "problem list" is the variable field that allows reporting and tracking this diagnosis across visits. Maintaining an accurate problem list is also a core measure of EHR Meaningful Use. ${ }^{27}$ Next, for those patients without a same-day problem list documentation of asthma, we estimated time from any diagnosis to problem list recording. We also considered a binary outcome of whether asthma severity was ever recorded on the problem list. Asthma severity was only reflected in diagnosis coding (ICD-10) after October 1, 2015, so evaluating documentation of asthma severity was performed in a subset of children with a visit after this date. For the set of prescription outcomes, we analyzed annual rates of albuterol, inhaled corticosteroid, and oral corticosteroid prescriptions, all which are medications recommended in national guidelines, for daily control or to be prescribed or had on hand for exacerbations. ${ }^{13}$ Similarly, as inhaled and oral steroids are more commonly indicated for children with persistent asthma, ${ }^{13}$ we also used this visit restriction along with a requirement that patients have documented persistent asthma during this time. Analyses of albuterol were done on our entire sample with asthma.

\section{Variables}

Our primary independent variables were a combination of ethnicity and preferred language expressed as 3 groups: non-Hispanic white patients, Spanish-preferring Latino patients, and English-preferring Latino patients. While we use Latino/a because it is often preferred in our study population, the actual ethnicity information collected by clinics is Hispanic and non-Hispanic white. 


\section{Covariates}

Patient characteristics were derived from the date of first asthma diagnosis in our record including age, sex, insurance status at first diagnosis, family income as measured by percent of federal poverty level, and BMI. In addition, we estimated the rate of ambulatory visits (for any reason) per year up to and including the first asthma diagnosis and included it as a covariate.

\section{Statistical Analysis}

We described patient characteristics in our total sample and between our 3 ethnicity/language groups. For the binary outcome of whether asthma was documented on the problem list the same day as diagnosis, we used generalized estimating equation (GEE) logistic regression model to estimate odds ratios comparing ethnicity/language groups adjusted for covariates. We also performed a similar GEE logistic model for the binary outcome of whether asthma severity was ever documented. We fitted GEE logistic models with a compound symmetry correlation structure and empirical sandwich variance estimator to account for clustering of patients by clinic.

For those patients who did not have asthma recorded on the problem list on the day of first diagnosis, we utilized state-stratified Cox regression with Breslow method for ties to assess ethnicity/language differences in time-to-problem-list-record from the date of first diagnosis, adjusted for the same covariates. A robust sandwich estimator was used to construct 95\% CIs for the hazard ratios (HRs), accounting for the clustering of patients by clinic. The assumption of proportional hazards was assessed using Schoenfeld residuals and was deemed suitable.

For the set of analyses evaluating annual prescription rates of common asthma medications, we first provide unadjusted annual prescription rate estimates for each medication by ethnicity/language group. Next, for albuterol, corticosteroid inhalers, and oral steroids separately, we estimate using a Poisson-logit hurdle regression model ${ }^{30}$ for both 1) the odds of receiving any prescription in the study period by ethnicity/language groups, and 2) among those with a prescription (ie, those who passed the hurdle), the rate ratios of prescriptions by ethnicity/ language groups. Similar to zero-inflated Poisson models, these Poisson-logit hurdle regressions model 2 processes. The first process examines the decision to prescribe a medication or not (modeled through a logit model) and in the second process, for patients who were prescribed a medication, a Poisson model is used to evaluate the rates of prescriptions over the study period. For all models, we adjusted for the covariates listed above. In the albuterol analysis, we included an additional covariate for patients' maximum recorded level of asthma-severity (mild intermittent, mild persistent, moderate persistent, severe persistent, or not documented). In the corticosteroid inhaler and oral steroids models, we also included an additional covariate for maximum level of persistent asthma on record (mild, moderate, or severe persistent). All hurdle models utilized robust sandwich variance estimation to account for clustering of patients by clinic. All statistical tests were performed with a 2-sided type I error of 5\%. Analyses were conducted in Stata version 15 (StataCorp, College Station, TX). This study was approved by the Institutional Review Board of Oregon Health and Science University.

\section{Results}

Descriptive statistics for the study population and by ethnicity/language groups are included in Table 1. Of note, Latino patients (Spanish and English speaking) had a higher prevalence of Medicaid insurance, a higher proportion of individuals under $138 \%$ of the federal poverty level, and a higher proportion of obese individuals than non-Hispanic white children. Spanish-speaking children had a slightly higher proportion of Medicaid insurance, and non-Hispanic white children had a higher proportion of private insurance. Additional descriptive statistics, by cohort, of the subpopulation of patients with a visit after October 1, 2015 (our denominator for those who could have ICD-10 documentation of asthma severity) is included in Appendix Table 1. Appendix Table 2 compares the characteristics of patients with a visit after October 1, 2015, to those without a visit after that date.

Figure 1 demonstrates our findings related to the documentation of asthma as a chronic condition. Latino patients (Spanish and English-preferring) had lower odds than non-Hispanic whites of having their asthma recorded on their problem list on the first day the diagnosis was noted (odds ratio $[\mathrm{OR}]=0.83$; $95 \% \mathrm{CI}, 0.77$ to 0.89 for Spanish preferring; $\mathrm{OR}=0.93 ; 95 \% \mathrm{CI}, 0.87$ to 0.99 for English preferring). If it was not diagnosed on the first day, however, Spanish-preferring Latino 
Table 1. Study Patient Characteristics, Overall and by Ethnicity/Language Groups

\begin{tabular}{|c|c|c|c|c|}
\hline \multirow[b]{3}{*}{ Characteristic } & \multirow[b]{3}{*}{ All Patients, N (\%) } & \multicolumn{3}{|c|}{ Ethnicity/Language Group, N (\%) } \\
\hline & & $\underline{\text { Non-Hispanic }}$ & Latino & Latino \\
\hline & & White & Preferring Spanish & Preferring English \\
\hline Patients, N (row \%) & $37,614(100.0)$ & $12,747(33.9)$ & $13,472(35.8)$ & $11,395(30.3)$ \\
\hline Female & $16,450(43.7)$ & $5688(44.6)$ & $5756(42.7)$ & $5006(43.9)$ \\
\hline Age in years, mean (SD) & $8.0(4.0)$ & $8.6(4.1)$ & $7.4(3.8)$ & $8.0(4.0)$ \\
\hline \multicolumn{5}{|l|}{ Payor type } \\
\hline Private & $3372(9.0)$ & $1975(15.5)$ & $421(3.1)$ & $976(8.6)$ \\
\hline Medicaid & $30,552(81.2)$ & $9637(75.6)$ & $11,523(85.5)$ & $9392(82.4)$ \\
\hline Medicare & $34(0.1)$ & $23(0.2)$ & $3(0.0)$ & $8(0.1)$ \\
\hline Other public & $379(1.0)$ & $116(0.9)$ & $152(1.1)$ & $111(1.0)$ \\
\hline Uninsured & $3277(8.7)$ & $996(7.8)$ & $1373(10.2)$ & $908(8.0)$ \\
\hline \multicolumn{5}{|l|}{ Federal poverty level } \\
\hline$\leq 138 \%$ & $23,184(61.6)$ & $7248(56.9)$ & $9067(67.3)$ & $6869(60.3)$ \\
\hline$>138 \%$ & $3412(9.1)$ & $1467(11.5)$ & $991(7.4)$ & $954(8.4)$ \\
\hline No information & $11,018(29.3)$ & $4032(31.6)$ & $3414(25.3)$ & $3572(31.3)$ \\
\hline \multicolumn{5}{|l|}{ Body mass index* } \\
\hline Normal & $20,589(54.7)$ & $7547(59.2)$ & $6964(51.7)$ & $6078(53.3)$ \\
\hline Overweight & $6385(17.0)$ & $2077(16.3)$ & $2347(17.4)$ & $1961(17.2)$ \\
\hline Obese & $10,640(28.3)$ & $3123(24.5)$ & $4161(30.9)$ & $3356(29.5)$ \\
\hline Yearly visits $^{\dagger}$, mean (SD) & $2.9(2.6)$ & $2.6(2.4)$ & $3.3(2.7)$ & $2.9(2.7)$ \\
\hline
\end{tabular}

Note: All patient characteristics were derived at first asthma diagnosis at age 3 years or older unless otherwise specified.

Column p percentages are reported unless otherwise stated.

*Body mass index (BMI) calculated using the 'childsds' package in R based on age, sex, weight, and height. Overweight if BMI over the 85 th percentile, obese if over the 95 th percentile. Biologically implausible values were flagged as over 8 standard deviations over or 4 under the mean BMI.

${ }^{\dagger}$ Yearly visits for any reason up to and including first asthma diagnosis at age 3 years or older.

$\mathrm{SD}$, standard deviation.

Figure 1. Measures of problem list documentation outcomes among children (ages 3 to 17 years) with asthma comparing Latino English-preferring and Latino Spanish-preferring to non-Hispanic whites across 21 US states, 2005 to 2017.

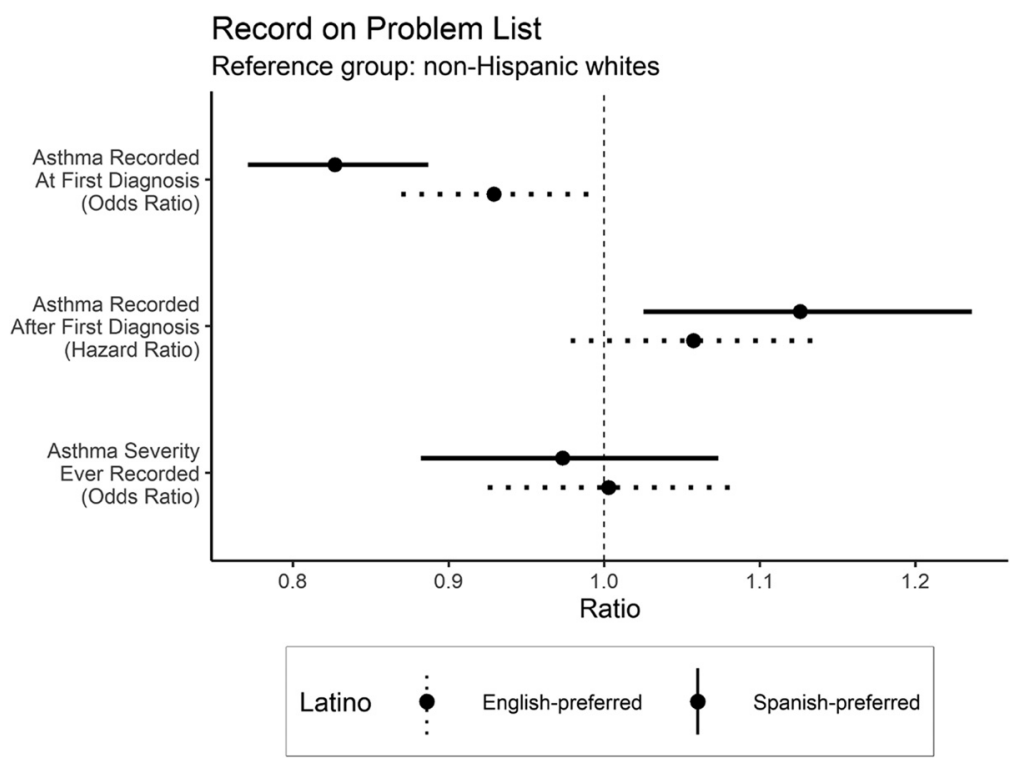


Table 2. Unadjusted Yearly Prescription Rates for Latino and non-Hispanic White Children (Age 3-17 Years) Diagnosed with Asthma and Having at Least One Ambulatory Visit in the OCHIN or Health Choice Network, 20052017

\begin{tabular}{lccc}
\hline Drug Class & $\begin{array}{c}\text { Non-Hispanic White Rate } \\
(95 \% \text { CI })\end{array}$ & $\begin{array}{c}\text { Hispanic Spanish-Preferred Rate } \\
(95 \% \text { CI })\end{array}$ & $\begin{array}{c}\text { Hispanic English-Preferred Rate } \\
(95 \% \text { CI })\end{array}$ \\
\hline Albuterol & $1.27(1.20,1.33)$ & $1.35(1.24,1.45)$ & $1.34(1.19,1.49)$ \\
Corticosteroid Inhaler* & $1.22(1.05,1.40)$ & $1.22(0.94,1.49)$ & $1.00(0.62,1.37)$ \\
$\quad$ Mild-persistent & $1.12(0.92,1.31)$ & $1.22(0.91,1.53)$ & $0.90(0.41,1.38)$ \\
$\quad$ Moderate-severe persistent & $1.36(1.16,1.57)$ & $1.21(0.94,1.47)$ & $1.21(0.99,1.44)$ \\
Oral steroid* & $0.49(0.40,0.57)$ & $0.47(0.40,0.54)$ & $0.47(0.35,0.59)$ \\
Mild-persistent & $0.42(0.29,0.54)$ & $0.40(0.33,0.47)$ & $0.36(0.25,0.47)$ \\
Moderate-severe persistent & $0.58(0.49,0.67)$ & $0.60(0.51,0.69)$ & $0.69(0.57,0.81)$ \\
\hline
\end{tabular}

*Rates for children with persistent-asthma on record, overall and stratified by persistent-level. CI, confidence interval.

patients had their asthma diagnosis recorded sooner than non-Hispanic white children ( $\mathrm{HR}=1.13 ; 95 \%$ CI, 1.03 to 1.24). English-speaking Latino patients showed similar time to problem list documentation as non-Hispanic white children ( $\mathrm{HR}=1.05 ; 95 \%$ CI, 0.98 to 1.14). There was no difference between ethnicity/language groups in EHR problem list documentation of asthma severity $(\mathrm{OR}=0.95 ; 95 \%$ CI, 0.87 to 1.05 for Spanish-preferring Latinos; $\mathrm{OR}=0.99$; $95 \%$ CI, 0.92 to 1.06 for English-preferring Latinos).

Unadjusted prescription rates of the 3 ethnicity/ language groups are shown in Table 2. Generally, children in our study population had similar unadjusted annual rates of common asthma medications.

Figure 2 demonstrates the adjusted relative odds of ever having received a prescription of albuterol or inhaled or oral steroids. Spanish-preferring Latino patients had significantly higher odds of ever receiving a prescription for albuterol than non-Hispanic white children $(\mathrm{OR}=1.96 ; 95 \% \mathrm{CI}$, 1.52 to 2.52). Similarly, Spanish-preferring Latino patients had higher odds of ever having received a prescription for corticosteroids ( $\mathrm{OR}=1.45 ; 95 \%$ CI, 1.01 to 2.09 ) and oral steroids $(\mathrm{OR}=1.48 ; 95 \%$ CI, 1.07 to 2.04) compared with non-Hispanic white children. This association for corticosteroids and oral steroids was also observed among children with mild-persistent asthma when stratified by asthma severity. We observed no differences in the odds of receiving any corticosteroids or oral steroids prescription between Spanish-preferring Latinos and non-Hispanic white children with moderate to severe persistent asthma. Comparing
English-preferring Latino patients to non-Hispanic white patients, we saw similar odds of prescriptions of albuterol, or inhaled or oral steroids, overall and by level of severity.

Figure 3 displays, among children with a prescription during the study period, the adjusted rate ratios (aRRs) of common asthma prescriptions by our defined ethnicity/language groups. Of note, Spanish-preferring Latino children had higher rates of albuterol prescriptions compared with nonHispanic whites (RR = 1.07; 95\% CI, 1.01 to 1.13 ). Overall, Spanish-preferring Latinos and nonHispanic white children had similar prescription rates of inhaled corticosteroid and oral steroid. However, among the sample of patients with mild persistent asthma severity, we observed that Spanish-preferring patients receive higher rates of inhaled corticosteroid ( $\mathrm{RR}=1.18$; 95\% CI, 1.04 to $1.34)$, but lower rates of oral steroids $(\mathrm{RR}=0.73$; 95\% CI, 0.55 to 0.98 ) compared with non-Hispanic white children with asthma. There were no other differences in common asthma prescription rate between English-preferring Latinos and nonHispanic whites.

\section{Discussion}

This analysis of care quality measures for asthma in Latino and non-Hispanic white children is novel in its sample size, use of longitudinal EHR data, and ability to consider language while accounting for numerous clinical and sociodemographic features. Spanish-speaking Latino children were less likely than our other cohorts to have the diagnosis of 
Figure 2. Estimates of relative odds of ever receiving specific prescription medications comparing Latino Englishpreferring and Latino Spanish-preferring to non-Hispanic white children across 21 US states, 2005 to 2017. For corticosteroid inhalers and oral steroids outcomes, we report odds ratios overall (among all patients with persistent asthma) and stratified by maximum level of persistent asthma denoted by parentheses.

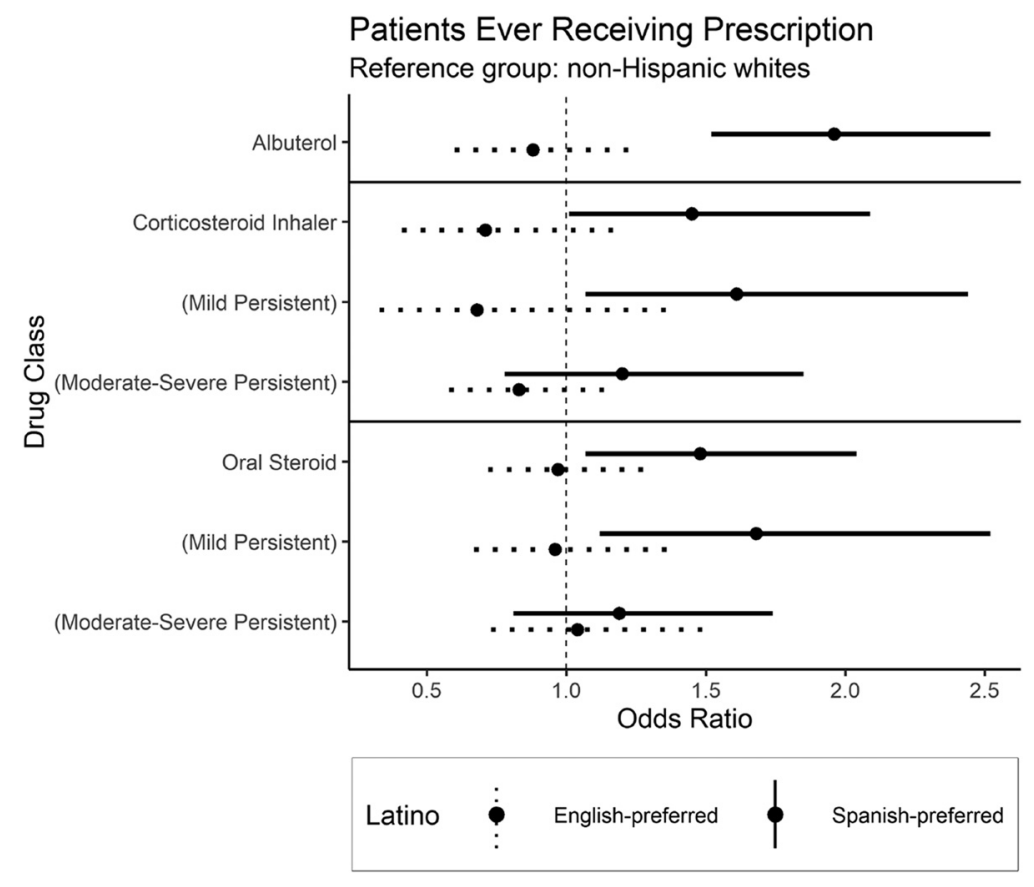

asthma recorded on their problem list on the same day it was first noted in the chart, but the diagnosis of asthma was recorded more quickly after the first day. This pattern could represent an initial language-related barrier to the documentation of asthma as a chronic problem, but a more rapid follow up-possibly initiated by family or providerthat leads to problem list documentation. Providers should continue to be cognizant of the need for rapid documentation/communication of asthma as a chronic problem once diagnosed. The documentation of asthma severity was equitable between the cohorts. This is crucial as asthma severity guides appropriate medication therapy, and equity in medication therapy depends on the appropriate documentation of asthma diagnosis.

Our analyses revealed the surprising finding that Spanish-speaking children had almost double the odds of having been prescribed albuterol and were more likely to have ever been prescribed inhaled or oral corticosteroids as well. In addition, among children prescribed medication, Spanish-speaking Latino children had higher rates of albuterol prescriptions, and when diagnosed with mild persistent asthma, had higher rates of inhaled and lower rates of oral corticosteroids. Overall, it is encouraging, that in this $\mathrm{CHC}$ population with established asthma diagnoses, Latino children (English and Spanish preferring) did not experience reduced prescription rates for common asthma medications. This suggests that any asthma disparities experienced by this population may occur at other stages of care (medication filling, initial diagnosis) rather than at the stage of appropriate prescribing to match asthma diagnosis/severity. We were not able to measure specific symptoms, presentations, or other factors which may bring about prescriptions, so it is uncertain whether Spanishspeaking children received more prescriptions because their asthma was somehow worse, although we did stratify by asthma severity when that documentation was appropriate. However, the specific pattern in Spanish-preferring children with mild persistent asthma of increased inhaled steroid prescriptions and decreased oral steroid prescriptions is consistent with more guideline concordant care. Spanish-preferring children may have more family, cultural, or community resources/support that aid in the management and followup of this chronic condition; however, our analysis was unable to measure these factors, and we could not assess overdiagnosis 
Figure 3. Among patients who were prescribed a medication, estimates of relative rates of specific prescription medications comparing Latino English-preferring and Latino Spanish-preferring to non-Hispanic whites across 21 US states, 2005 to 2017. For corticosteroid inhalers and oral steroids outcomes, we report odds ratios overall (among patients with persistent-asthma) and stratified by maximum level of persistent asthma denoted by parentheses.

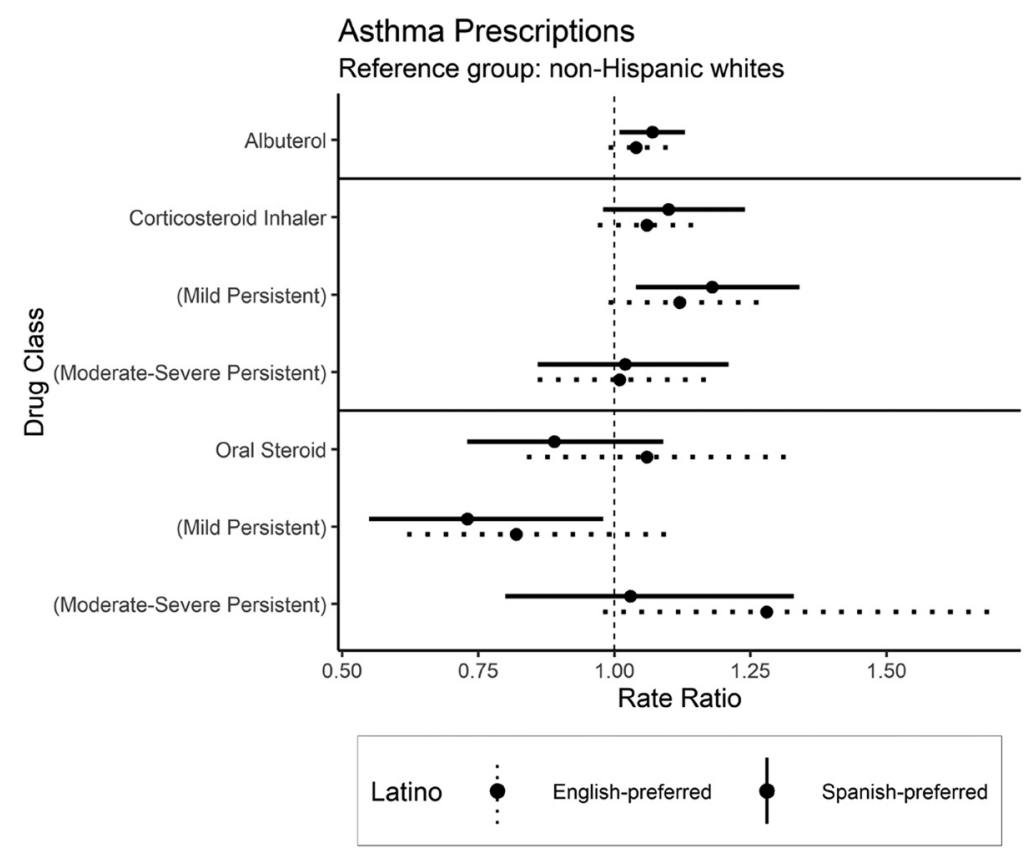

or over prescribing as a response to uncertainty in communication because of language barriers. Given the known high prevalence of asthma among Latino children and the documented barriers to medication use, CHC providers may be especially attentive to this condition in this population, and have a low threshold for the prescription of these medications, as literature does show that limited English proficiency can be a barrier to asthma medication use. ${ }^{31}$ Future studies can delve into the individual and provider factors (including language ability) that might have resulted in these findings in these children.

While previously observed findings by Kharat, ${ }^{3}$ Crocker, ${ }^{6}$ and $\mathrm{Kit}^{8}$ showed less inhaled steroid prescriptions in Latino children, our analysis was conducted using a much larger sample across 21 states, observed over a longer period of time, controlled for more covariates, examined a low-income population (which is therefore especially vulnerable to inequity) in objective EHR data, and stratified by asthma severity. Spanish- and English-speaking Latino children, with varying levels of overall health care utilization, health insurance, and socioeconomic status, received equitable asthma care—once diagnosed—on numerous asthma care indicators. Our findings may suggest that CHCs in our nation's safety net may provide a worthwhile template for reducing some disparities in asthma care. This has been shown in other disease processes in Latino patients. ${ }^{32}$ However, understanding the role and potential mechanisms $\mathrm{CHCs}$ play and how they can be utilized in reducing disparities requires further study.

\section{Limitations}

Our analysis was in a low-income population seen in CHCs and may not be generalizable to other populations. Our dataset did not include prescription-fill information or the quality of asthma education or risk factor assessment, which are important additional factors to consider in asthma care. We also did not have Hispanic subgroup information in this analysis, which some literature suggests may affect asthma course. ${ }^{33,34}$ However, the literature on Hispanic subgroup focuses mostly on asthma prevalence, and our analysis of care quality measures focuses on those already diagnosed making specific subgroup differences in prevalence less relevant here. Applying this analysis to subgroups could represent a direction for further research. Provider language or quality of interpreters were also not assessed in this analysis-we did not have access to 
provider language ability, although all $\mathrm{CHCs}$ are required to have language-appropriate services, ${ }^{35}$ so ideal standards are equivalent across our study clinics. Future analyses can also study how quickly children receive follow-up care for asthma after diagnosis. We did not analyze non-Hispanic black children, as disparities in Latino children potentially encompass distinct issues from those faced in African American communities: geographic differences, immigration barriers, possible insurance differences, language barriers, and possible cultural factors, among others. Therefore, a specific examination of possible disparities in Latino children, compared with a single dominant culture majority, would seem warranted to focus on this population and its unique set of health care experiences. A proper analysis of black children was simply outside the scope of this article. Lastly, it is possible that children in this study received care outside of ADVANCE national CHC network; however, in previous work we have shown that patients are unlikely to leave the network. ${ }^{36}$ Finally, we were unable to tell if oral steroids were prescribed in direct response to an asthma exacerbation or if they were given to have on hand for children with more severe disease or frequent exacerbations. Future work can explore this specific scenario further.

\section{Conclusion}

In a national network of CHCs, Latino children had lower odds of having their asthma diagnosis recorded on their problem list on the same day the diagnosis was noted as compared with non-Hispanic white children, but had it recorded more quickly after the first day. Spanish-preferring children were more likely to have ever been prescribed albuterol, inhaled or oral corticosteroids, and among those with any prescriptions, had higher rates of albuterol prescriptions. When diagnosed with mild persistent asthma, Spanish-preferring Latino children had higher rates of inhaled and lower rates of oral corticosteroids. In many of the care measures we examined, there was no difference in the care documented between non-Hispanic white children, Spanish-preferring Latino children, and Englishpreferring Latino children, which may help in directing further research toward other aspects of the asthma care continuum. Further research should examine medication fill/use rates, presenting symptoms and correlating prescriptions, initial diagnosis rates among children with symptoms, and how CHCs may be a model for improving health equity.

The research reported in this manuscript was conducted with the Accelerating Data Value Across a National Community Health Center Network (ADVANCE) Clinical Research Network, a partner of PCORnet ${ }^{\circledR}$, the National-Patient Centered Clinical Network, an initiative of the Patient Centered outcomes Research Institute (PCORI). The ADVANCE network is led by OCHIN in partnership with the Health Choice Network, Fenway Health, Oregon Health and Science University, and the Robert Graham Center/ HealthLandscape. ADVANCE is funded through PCORI award number 13-060-4716. We would also like to acknowledge Ms. Roopradha Datta, who assisted in manuscript preparation.

To see this article online, please go to: http://jabfm.org/content/ 33/5/707.full.

\section{References}

1. Centers for Disease Control and Prevention. Most Recent Asthma Data. https://www.cdc.gov/asthma/ most_recent_data.htm. Accessed March 4, 2020.

2. Wu AC, Butler MG, Li L, et al. Primary adherence to controller medications for asthma is poor. Annals ATS 2015;12:161-6.

3. Kharat AA, Borrego ME, Raisch DW, Roberts MH, Blanchette CM, Petersen H. Assessing disparities in the receipt of inhaled corticosteroid prescriptions for asthma by Hispanic and non-Hispanic white patients. Annals ATS 2015;12:174-83.

4. Bruzzese JM, Stepney C, Fiorino EK, et al. Asthma self-management is sub-optimal in urban Hispanic and African American/black early adolescents with uncontrolled persistent asthma. J Asthma 2012;49:90-7.

5. Carlin C, Yee AB, Fagnano M, Halterman JS. The influence of Hispanic ethnicity on parent-provider communication about asthma. Clin Pediatr (Phila) 2014;53:380-6.

6. Crocker D, Brown C, Moolenaar R, et al. Racial and ethnic disparities in asthma medication usage and health-care utilization: data from the National Asthma Survey. Chest 2009;136:1063-71.

7. Jandasek B, Ortega AN, McQuaid EL, et al. Access to and use of asthma health services among Latino children: the Rhode Island-Puerto Rico asthma center study. Med Care Res Rev 2011;68:683-98.

8. Kit BK, Simon AE, Ogden CL, Akinbami LJ. Trends in preventive asthma medication use among children and adolescents, 1988-2008. Pediatrics 2012;129:62-9.

9. Ortega AN, Gergen PJ, Paltiel AD, Bauchner H, Belanger KD, Leaderer BP. Impact of site of care, race, and Hispanic ethnicity on medication use for childhood asthma. Pediatrics 2002;109:E1.

10. Sarpong EM, Miller GE. Racial and ethnic differences in childhood asthma treatment in the United States. Health Serv Res 2013;48:2014-36. 
11. Stevens GD, Pickering TA, Seid M, Tsai KY. Disparities in the national prevalence of a quality medical home for children with asthma. Acad Pediatr 2009;9:234-41.

12. Stewart KA, Higgins PC, McLaughlin CG, Williams TV, Granger E, Croghan TW. Differences in prevalence, treatment, and outcomes of asthma among a diverse population of children with equal access to care: findings from a study in the military health system. Arch Pediatr Adolesc Med 2010;164:720-6.

13. National Heart Lung and Blood Institute Expert Panel Report 3: Guidelines for the Diagnosis and Management of Asthma. Bethesda, MD: National Heart, Lung, and Blood Institute (US); 2007.

14. National Institute of Diabetes and Digestive and Kidney Diseases. Chronic Care Model. Available from: https://www.niddk.nih.gov/health-information/ communication-programs/ndep/health-professionals/ practice-transformation-physicians-health-care-teams/ team-based-care/chronic-care-model.

15. Coleman K, Austin BT, Brach C, Wagner EH. Evidence on the Chronic Care Model in the new millennium. Health Aff (Millwood) 2009;28:75-85.

16. McDaniel MK, Waldfogel J. Racial and ethnic differences in the management of childhood asthma in the United States. J Asthma 2012;49:785-91.

17. Ojha RP, Tota JE, Offutt-Powell TN, Klosky JL, Ashokkumar R, Gurney JG. The accuracy of human papillomavirus vaccination status based on adult proxy recall or household immunization records for adolescent females in the United States: results from the National Immunization Survey-Teen. Ann Epidemiol 2013;23:281-5.

18. Mojica CM, Bastani R. Receipt of diagnostic tests for breast cancer: validity of self-reports among low-income, mostly Latina, indigent women. Eval Health Prof 2010;33:437-51.

19. Miles M, Ryman TK, Dietz V, Zell E, Luman ET. Validity of vaccination cards and parental recall to estimate vaccination coverage: a systematic review of the literature. Vaccine 2013;31:1560-8.

20. Fiscella K, Holt K, Meldrum S, Franks P. Disparities in preventive procedures: comparisons of self-report and Medicare claims data. BMC Health Serv Res 2006;6:122.

21. Clark NM, Dodge JA, Shah S, Thomas LJ, Andridge RR, Awad D. A current picture of asthma diagnosis, severity, and control in a low-income minority preteen population. J Asthma 2010;47:150-5.

22. National Association of Community Health Centers. Community Health Center Chartbook. 2019. Available from: http://www.nachc.org/wp-content/uploads/ 2019/01/Community-Health-Center-ChartbookFINAL-1.28.19.pdf.

23. DeVoe JE, Gold R, Cottrell E, et al. The ADVANCE network: accelerating data value across a national community health center network. JAMIA 2014;21:591-5.

24. BMJ Best Practice. Asthma in Children. 2018. Available from: https://bestpractice.bmj.com/topics/ en-us/782.

25. Black MH, Smith N, Porter AH, Jacobsen SJ, Koebnick C. Higher prevalence of obesity among children with asthma. Obesity 2012;20:1041-7.

26. Vo P, Makker K, Matta-Arroyo E, Hall CB, Arens R, Rastogi D. The association of overweight and obesity with spirometric values in minority children referred for asthma evaluation. JAsthma 2013;50:56-63.

27. Center for Medicare Services. Eligible Professional Meaningful Use Core Measures Measure 3 of 13. 2014. Available from: https://www.cms.gov/ Regulations-and-Guidance/Legislation/EHRIncentive Programs/downloads/3_Maintain_Problem_ListEP. pdf.

28. Wright A, McCoy AB, Hickman TTT, et al. Problem list completeness in electronic health records: A multi-site study and assessment of success factors. Int J Med Inform 2015;84:784-90.

29. Jolly SE, Navaneethan SD, Schold JD, et al. Chronic kidney disease in an electronic health record problem list: quality of care, ESRD, and mortality. Am J Nephrol 2014;39:288-96.

30. Loeys T, Moerkerke B, De Smet O, Buysse A. The analysis of zero-inflated count data: beyond zeroinflated Poisson regression. Br J Math Stat Psychol 2012;65:163-80.

31. McQuaid EL. Barriers to medication adherence in asthma: the importance of culture and context. Ann Allergy Asthma Immunol 2018;121:37-42.

32. Sadowski D, Devlin M, Hussain A. Better care at safety net providers? Utilization of recommended standards of diabetes care for rural Latinos in one Midwestern state. J Health Care Poor Underserved 2011;22:995-1013.

33. Arcoleo KJ, McGovern C, Kaur K, et al. Longitudinal patterns of Mexican and Puerto Rican children's asthma controller medication adherence and acute healthcare use. Annals ATS 2019;16:715-23.

34. Camacho-Rivera M, Kawachi I, Bennett GG, Subramanian SV. Revisiting the Hispanic health paradox: the relative contributions of nativity, country of origin, and race/ethnicity to childhood asthma. J Immigrant Minority Health 2015;17:826-33.

35. Cornell Law School. Public Health Services Act: 42 U.S. Code 254b - Health Centers. Available from: https://www.law.cornell.edu/uscode/text/42/254b.

36. O'Malley JP, O'Keeffe-Rosetti M, Lowe RA, et al. Health care utilization rates after Oregon's 2008 Medicaid expansion: within-group and betweengroup differences over time among new, returning, and continuously insured enrollees. Med Care 2016;54:984-91. 
Appendix Table 1. Characteristics at First Asthma Diagnosis Age 3 Years or Older for Patients Age 3-17 Years Having Had at Least 1 Ambulatory Visit in the OCHIN and Health Choice Network Networks on or after October 1, 2015

\begin{tabular}{|c|c|c|c|c|}
\hline \multirow[b]{3}{*}{ Characteristic } & \multirow[b]{3}{*}{ All Patients, No. (\%) } & \multicolumn{3}{|c|}{ Cohort, No. (\%) } \\
\hline & & $\underline{\text { Non-Hispanic }}$ & Hispanic & Hispanic \\
\hline & & White & Prefer Spanish & Prefer English \\
\hline Patients & 31,195 & $9988(32.0)$ & $11,825(37.9)$ & $9382(30.1)$ \\
\hline Female & $13,816(44.3)$ & $4576(45.8)$ & $5085(43.0)$ & $4155(44.3)$ \\
\hline Age, mean (SD), y & $8.1(4.1)$ & $8.8(4.2)$ & $7.4(3.8)$ & $8.0(4.1)$ \\
\hline \multicolumn{5}{|l|}{ Payor type } \\
\hline Private & $2706(8.7)$ & $1565(15.7)$ & $346(2.9)$ & $795(8.5)$ \\
\hline Medicaid & $25,647(82.2)$ & $7617(76.3)$ & $10212(86.4)$ & $7818(83.3)$ \\
\hline Medicare & $29(0.1)$ & $20(0.2)$ & $3(0.0)$ & $6(0.1)$ \\
\hline Other public & $272(0.9)$ & $86(0.9)$ & $111(0.9)$ & $75(0.8)$ \\
\hline Uninsured & $2541(8.1)$ & $700(7.0)$ & $1153(9.8)$ & $688(7.3)$ \\
\hline \multicolumn{5}{|l|}{ Federal poverty level } \\
\hline$\leq 138 \%$ & $19,097(61.2)$ & $5641(56.5)$ & $7879(66.6)$ & $5577(59.4)$ \\
\hline$>138 \%$ & $2802(9.0)$ & $1182(11.8)$ & $854(7.2)$ & $766(8.2)$ \\
\hline No information & $9296(29.8)$ & $3165(31.7)$ & $3092(26.1)$ & $3039(32.4)$ \\
\hline \multicolumn{5}{|l|}{ Body mass index* } \\
\hline Normal & $16,998(54.5)$ & $5908(59.2)$ & $6107(51.6)$ & $4983(53.1)$ \\
\hline Overweight & $5288(17.0)$ & $1593(15.9)$ & $2072(17.5)$ & $1623(17.3)$ \\
\hline Obese & 8909 (28.6) & $2487(24.9)$ & $3646(30.8)$ & $2776(29.6)$ \\
\hline Yearly visits $^{\dagger}$, mean (SD) & $3.1(2.7)$ & $2.7(2.5)$ & $3.4(2.7)$ & $3.0(2.8)$ \\
\hline
\end{tabular}

*Body mass index (BMI) calculated using the 'childsds' package in R based on age, sex, weight, and height. Overweight if BMI over the 85 th percentile, obese if over the 95 th percentile. Biologically implausible values were flagged as over 8 standard deviations over or 4 under the mean BMI.

${ }^{\dagger}$ Yearly Visits up to and including first asthma diagnosis at age 3 years or older.

SD, standard deviation; OCHIN, Oregon Community Health Information Network. 
Appendix Table 2. Characteristics at First Asthma Diagnosis Age 3 Years or Older for Patients Age 3-17 Years Having Had at Least 1 Ambulatory Visit in the OCHIN and Health Choice Network Networks, Comparing Those with and without a Visit after October 1, 2015

\begin{tabular}{|c|c|c|c|}
\hline Characteristic & All Patients, No. (\%) & $\begin{array}{c}\text { Had a Visit on/after } \\
\text { October 1, 2015, No. (\%) }\end{array}$ & $\begin{array}{l}\text { Did Not Have a Visit on/after } \\
\text { October 1, 2015, No. (\%) }\end{array}$ \\
\hline Patients & 37,614 & $31,195(83)$ & $6419(17)$ \\
\hline Female & $16,450(43.7)$ & $13,816(44.3)$ & $2634(41.0)$ \\
\hline Age, mean (SD), y & $8.0(4.0)$ & $8.1(4.1)$ & $7.6(3.4)$ \\
\hline \multicolumn{4}{|l|}{ Payor type } \\
\hline Private & $3372(9.0)$ & $2706(8.7)$ & $666(10.4)$ \\
\hline Medicaid & $30,552(81.2)$ & $25,647(82.2)$ & 4905 (76.4) \\
\hline Medicare & $34(0.1)$ & $29(0.1)$ & $5(0.1)$ \\
\hline Other public & $379(1.0)$ & $272(0.9)$ & $107(1.7)$ \\
\hline Uninsured & $3277(8.7)$ & $2541(8.1)$ & $736(11.5)$ \\
\hline \multicolumn{4}{|l|}{ Federal poverty level } \\
\hline$\leq 138 \%$ & $23184(61.6)$ & $19097(61.2)$ & $4087(63.7)$ \\
\hline$>138 \%$ & $3412(9.1)$ & $2802(9.0)$ & $610(9.5)$ \\
\hline No information & $11018(29.3)$ & $9296(29.8)$ & $1722(26.8)$ \\
\hline \multicolumn{4}{|l|}{ Body mass index* } \\
\hline Normal & $20589(54.7)$ & $16998(54.5)$ & $3591(55.9)$ \\
\hline Overweight & $6385(17.0)$ & $5288(17.0)$ & $1097(17.1)$ \\
\hline Obese & $10640(28.3)$ & 8909 (28.6) & $1731(27.0)$ \\
\hline Yearly visits $^{\dagger}$, mean $(\mathrm{SD})$ & $2.9(2.6)$ & $3.1(2.7)$ & $2.3(2.1)$ \\
\hline
\end{tabular}

The Patients in This Table Had a Valid BMI at First Asthma Diagnosis. Of the 49,057 Patients with Asthma 11,443 Did Not Have This Required BMI Data (9008 Had a Visit after Oct 1, 2015, and 2435 Did Not.

*Body mass index (BMI) calculated using the 'childsds' package in R based on age, sex, weight, and height. Overweight if BMI over the 85 th percentile, obese if over the 95 th percentile. Biologically implausible values were flagged as over 8 standard deviations over or 4 under the mean BMI.

${ }^{\dagger}$ Yearly visits up to and including first asthma diagnosis at age 3 years or older.

$\mathrm{SD}$, standard deviation; OCHIN, Oregon Community Health Information Network. 